María Nieves Carmona

Tello'

Michele Hernández

Cabrera $^{2}$

Eduardo Jerez Olivera ${ }^{3}$

Margarita Bolaños Rivero'

\title{
Canaliculitis por Mycobacterium abscessus subsp. abscessus
}

'Servicio de Microbiología y Parasitología Clínica, Hospital Universitario Insular de Gran Canaria.

${ }^{2}$ Unidad de Enfermedades Infecciosas y Medicina Tropical, Hospital Universitario Insular de Gran Canaria.

${ }^{3}$ Servicio de Oftalmología, Hospital Universitario Insular de Gran Canaria.

Article history

Received: 2 October 2020; Revision Requested: 23 October 2020; Revision Received: 10 No-vember 2020; Accepted: 23 November 2020; Published: 28 January 2021

Estimado Editor: la canaliculitis es la inflamación del canalículo, canal corto proximal en la vía lagrimal cerca del ángulo interno del párpado a través del cual drenan las lágrimas al saco lagrimal. La causa más frecuente es la infección por Actinomyces israelii pero puede ser ocasionada por otras bacterias, hongos o virus.

La colocación de material extraño en los canalículos para el tratamiento del ojo seco puede ser la causa de enfermedad debido a que existen bacterias capaces de formar biopelículas [1].

Referimos el caso de una paciente de 38 años con antecedentes de Tiroiditis de Hashimoto y Enfermedad de Behçet en tratamiento con metotrexato y ciclosporina tópica, que requirió de la colocación de tapones de silicona en los puntos lagrimales para el tratamiento del ojo seco.

Ante la inflamación del punto lagrimal y del canalículo inferior en el ojo izquierdo, sugestiva de canaliculitis, se procede a la dilatación del punto lagrimal y a la expresión del canalícuIo, con salida de material purulento acompañado de un tapón lagrimal que habría migrado al mismo. Se realizan lavados de la vía lagrimal y se pautan colirios de penicilina cada 6 horas.

Las muestras obtenidas se envían al Servicio de Microbiología y se siembran en los medios de cultivo habituales. A los 4 días de incubación, se detecta el crecimiento de unas colonias con aspecto rugoso y color cremoso mate que al gram se observan como bacilos grampositivos y en la tinción de Ziehl-Neelsen como bacilos ácido alcohol resistentes.

La especie se identifica mediante espectrometría de masas MALDI-TOF MS (Bruker $\left.{ }^{\circledR}\right)$ como Mycobacterium abscessus subsp. abscessus con un score de 2,03 y se confirma mediante PCR del gen 23s rRNA (GenoType ${ }^{\circledR}$ NTM-DR, Hain Lifescience).

Correspondencia:

Margarita Bolaños Rivero.

Servicio de Microbiologia y Parasitología Clínica, Hospital Universitario Insular de Gran

Canaria. Avda. Maritima del Sur, s/n, 35016, Gran Canaria, España.

Tfno: 928441763

Fax: 928441861

E-mail:mbolriv@gobiernodecanarias.org
El estudio de sensibilidad se realizó mediante E-test (Biomerieux ${ }^{\circledR}$ ) en Mueller Hinton Medium resultando sensible a claritromicina, linezolid, tobramicina, amikacina e imipenem.

La cepa se envió al Centro Nacional de Microbiología donde se confirmó su identificación y su sensibilidad a los antimicrobianos.

Con el resultado del cultivo se volvió a valorar a la paciente y se decide suspender el colirio de penicilina para comenzar un tratamiento especifico de 6 semanas con claritromicina y linezolid.

Actualmente la paciente se encuentra asintomática.

Las micobacterias de crecimiento rápido (MCR) son bacilos pleomórficos, aerobios, inmóviles, intracelulares y resistentes a las condiciones ambientales. Están distribuidas mundialmente en el medio ambiente pudiendo causar enfermedad en caso de lesiones crónicas, procedimientos estéticos, inyecciones, heridas quirúrgicas o traumáticas con exposición al agua o a productos contaminados [2].

El grupo de Mycobacterium chelonae/Mycobacterium abscessus está formado por: $M$. chelonae, $M$. immunogenum, $M$. abscessus subsp. abscessus (M. abscessus), M. abscessus subsp. bolletii (M. massilense y M.bolletii) y M.salmoniphilum [3].

Aunque la identificación de las MCR mediante la espectrometría de masas MALDI-TOF MS (Bruker ${ }^{\circledR}$ ) se demuestra como una técnica sencilla, rápida y con una relación coste-efectiva adecuada y varios estudios realizados demuestran una identificación correcta en el 98,06\% de los aislados, existen estudios que sugieren la dificultosa diferenciación entre los grupos M. abscessus subsp. abscessus (M. abscessus) y M. abscessus subsp. bolletii (M. massilense y M.bolletii) debido a su estrecha relación filogenética [3-5].

El grupo de $M$. chelonae/M. abscessus es generalmente resistente a fluoorquinolonas y sensible a macrólidos, imipenem, amikacina y tigeciclina, que se usa como alternativa [6-8].

Queremos concluir destacando varios aspectos importan- 
tes para el correcto diagnóstico de estas micobacterias de crecimiento rápido como son:

-La importancia de la incubación prolongada de las placas procedentes de muestras de piel y tejidos blandos (incluyendo las muestras oculares) para el aislamiento de las mismas [9].

- La sospecha clínica no es habitual, por tanto, en caso de que los cultivos sean negativos, se debe sospechar el diagnóstico y solicitar cultivo de micobacterias [10].

- La adecuada identificación de las diferentes subespecies de este grupo mediante la espectrometría de masas, aunque se requiere su confirmación con la técnica de referencia.

\section{AGRADECIMIENTOS}

Agradecemos a la Dra. María Soledad Jiménez Pajares del Centro Nacional de Microbiología (ISCIII, Majadahonda, Madrid) por su colaboración en la caracterización molecular de la micobacteria.

\section{FINANCIACIÓN}

Los autores declaran que no han recibido financiación para la realización de este estudio.

\section{CONFLICTO DE INTERESES}

Los autores declaran no tener conflicto de intereses.

\section{BIBLIOGRAFÍA}

1. Brown-Elliot BA, Wallace RJ. Infecciones debidas a micobacterias no tuberculosas. In: Mandell GL, Douglas RG, Bennett JE, Dolin R. Mandell, Douglas y Bennett, Enfermedades Infecciosas: Principios y Práctica. 7th ed. Pensilvania. Editorial Médica Panamericana 2012; p. 3187-3194.

2. Alcaide F, Esteban J. Cutaneous and soft skin infections due to non-tuberculous mycobacteria. Enferm Infecc Microbiol Clin 2010; 28: 46-50. doi: 10.1016/s0213-005x(10)70008-2 PMID: 20172423.

3. Brown-Elliot BA, Wallace RJ. Mycobacterium: Clinical and Laboratory Characteristics of Rapidly Growing Mycobacteria. In: Jorgensen JH, Pfaller MA, Carroll KC, Funke G, Landry ML, Richter SS, Warnock DW. Manual of Clinical Microbiology. 11 th Edition. Washington. ASM Press 2015; p. 595-612.

4. Fernández-Esgueva $\mathrm{M}$, Fernández-Simon $\mathrm{R}$, Monforte-Cirac $M L$, López-Calleja Al, Fortuño B, Viñuelas-Bayon J. Use of MALDI-TOF MS (Bruker Daltonics) for identification of Mycobacterium species isolated directly from liquid medium. Enferm Infecc Microbiol Clin 2020. doi: 10.1016/j. eimc.2020.05.011.

5. Saleeb PG, Drake SK, Murray PR, Zelazny AM. Identification of mycobacteria in solid-culture media by matrix-assisted laser desorption ionization-time of flight mass spectrometry. J Clin Microbiol 2011; 49: 1790-1794. doi:10.1128/ JCM.02135-10.

6. García-Martos P, García-Agudo L. Infecciones por micobacterias de crecimiento rápido. Enferm Infecc Microbiol Clin 2012; 30: 192-200. doi: 10.1016/j.eimc.2011.09.017.

7. Brown-Elliott BA, Mann LB, Hail D, Whitney C, Wallace Jr RJ. Antimicrobial susceptibility of nontuberculous mycobacteria from eye infections. Cornea 2012; 3: 900-906. doi: 10.1097/ICO.0b013e31823f8bb9.

8. Chu HS. Chang SC, Shen EP, Hu FR. Nontuberculous mycobacterial ocular infections-comparing the clinical and microbiological characteristics between Mycobacterium abscessus and Mycobacterium massiliense. PloS one 2015; 10: e0116236. doi: 10.1371/journal.pone.0116236.

9. Carrillo-Quintero D, Bolaños-Rivero M, Hernández-Cabrera $\mathrm{M}$, Cañas-Hernández F. Aislamiento de micobacterias de crecimiento rápido a partir de muestras de piel y tejidos blandos. Una etiología a tener en cuenta. Enferm Infecc Microbiol Clin 2014; 32: 692-693. doi: 10.1016/j. eimc.2014.03.005 .

10. López-Cerero L, Etxebarria J, Mensa J. Diagnóstico microbiológico de las infecciones oculares. Enferm Infecc Microbiol Clin 2009; 27: 531-535. doi: 10.1016/j. eimc.2009.01.006. 